\title{
Some properties on the homomorphism of groups
}

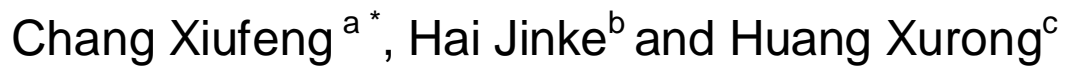 \\ School of Mathematics and Statistics, Qingdao University, Shandong 266071, P.R.China \\ a1018402779@qq.com, bhaijinke@qdu.edu.cn, ${ }^{c} 1376882875 @ q q . c o m$
}

Keywords: normal endomorphism; morphic endomorphism; morphic group; dual homomorphism

\begin{abstract}
In this paper, based on the basic of morphic groups and quasi-morphic groups, we defined a new kind of homomorphism with some properties, called dual homomorphism. We give a necessary and sufficient condition of the dual homomorphism. Moreover, we get a connection between the dual homomorphism and the structure of groups.
\end{abstract}

\section{Introduction}

W.K.Nicholson and M.F.Yousuf [1] have defined the concept of the morphic endomorphism, and applied this concept to the structure of ring. In 2010, Y.Li, W.K.Nicholson and L.Zan [2] applied the morphic endomorphism to the group structure, and gave some important connections between them. Q.Wang and K.Long [3] have generalized the concept of the morphic endomorphism on quasi-morphic, and showed that all finite abelian groups are quasi-morphic groups. In this paper, based on the basic of morphic groups and quasi-morphic groups, we continue to study a new group homomorphism with some properties, which is called dual homomorphism. Furthermore, we get that dual homomorphism is a generalization of morphic endomorphism, while it is different from quasi-morphic. And we studied some properties of dual homomorphism.

\section{Notations and Preliminaries}

In this paper, if $G$ is a group, we write $\operatorname{End}(G)$ for the set of endomorphism of $G$ and write $\operatorname{Aut}(G)$ for the group of automorphisms of $G$. Let $G_{1}$ and $G_{2}$ be groups, we write $\operatorname{Hom}\left(G_{1}, G_{2}\right)$ for the set of homomorphism of $G_{1}$ to $G_{2}$, and $I \operatorname{so}\left(G_{1}, G_{2}\right)$ for the isomorphism. If $\alpha \in \operatorname{End}(G)$, denote the image of group $G$ under the $\alpha$ by $G^{\alpha}$, and denote the kernel of $\alpha$ by $\operatorname{Ker}(\alpha)$. Moreover, other notations are mostly standard, please refer to [4].

Definition 2.1 Let $G_{1}$ and $G_{2}$ be groups, $\varphi \in \operatorname{Hom}\left(G_{1}, G_{2}\right)$ is called normal homomorphism if $G_{1}^{\varphi} \triangleleft G_{2}$. In particular, we call $\varphi$ is normal endomorphism of $G_{1}$ if $G_{1}=G_{2}$.

Definition 2.2 let $G$ be a group, $\alpha \in \operatorname{End}(G)$ is called morphic endomorphism if it satisfies the condition of $G^{\alpha} \triangleleft G$ and $G / G^{\alpha} \cong \operatorname{Ker}(\alpha)$. Moreover, $G$ is called morphic group if every normal endomorphism $\alpha$ of $G$ is morphic endomorphism.

Definition 2.3 let $G$ be a group, the normal endomorphism $\alpha$ of $G$ is called quasi-morphic if there exists normal endomorphism $\beta$ and $\gamma$ such that $\operatorname{Ker}(\alpha)=G^{\beta}$ and $G^{\alpha}=\operatorname{Ker}(\gamma)$.

Definition 2.4 Let $G_{1}$ and $G_{2}$ be groups, $\alpha \in \operatorname{Hom}\left(G_{1}, G_{2}\right)$ is called dual homomorphism if there exists $\beta \in \operatorname{Hom}\left(G_{2}, G_{1}\right)$ such that $\operatorname{Ker}(\alpha)=G_{2}{ }^{\beta}$ and $\operatorname{Ker}(\beta)=G_{1}^{\alpha}$. Moreover, we say that $G_{1}$ and $G_{2}$ are dual if every $\alpha \in \operatorname{Hom}\left(G_{1}, G_{2}\right)$ is dual homomorphism.

As a direct corollary of Definition 2.4, we have

Corollary 2.5 Assume $G_{1}$ and $G_{2}$ are dual, if $G_{1}=G_{2}$, then $G_{1}$ is a morphic group. 
Proof Suppose $\alpha \in \operatorname{Hom}\left(G_{1}, G_{2}\right)$, since $G_{1}$ with $G_{2}$ are dual, then $\alpha$ is a dual homomorphism. According to the definition of dual homomorphism that there exists $\beta \in \operatorname{Hom}\left(G_{2}, G_{1}\right)$ such that $\operatorname{Ker}(\alpha)=G_{2}{ }^{\beta}$ and $\operatorname{Ker}(\beta)=G_{1}^{\alpha}$, thus $G_{1}^{\alpha} \triangleleft G_{2}$ and $G_{2} / G_{1}^{\alpha} \cong \operatorname{Ker}(\alpha)$. By $G_{1}=G_{2}$, which implies that $G_{1}^{\alpha} \triangleleft G_{1}$ and $G_{1} / G_{1}^{\alpha} \cong \operatorname{Ker}(\alpha)$. Hence $\alpha$ is a morphic of $G_{1}$. So $G_{1}$ is a morphic group.

Proposition 2.6 Let $\alpha$ be a quasi-morphic of $G$, which means that there exist normal endomorphism $\beta$ and $\gamma$ such that $\operatorname{Ker}(\alpha)=G^{\beta}$ and $G^{\alpha}=\operatorname{Ker}(\gamma)$. If $\beta=\gamma$, then $\alpha$ is a dual homomorphism.

Lemma $2.7^{[4]}$ Let $K \triangleleft G$ and $\alpha \in \operatorname{Aut}(G)$. Write $K^{\alpha}=\left\{k^{\alpha} \mid k \in K\right\}$. Then $K \cong K^{\alpha} \triangleleft G$ and $G / K \cong G / K^{\alpha}$.

Lemma 2.8 ${ }^{[4]}$ Let $\varphi: G \rightarrow H$ be a homomorphism and let $K=\operatorname{Ker}(\varphi) \triangleleft G$. Let $\gamma$ be the natural homomorphism of $G$ onto $G / K$. Then there is an injective homomorphism $\psi: G / K \rightarrow H$ such that $\varphi=\gamma \psi$. In particular, $\operatorname{Im}(\varphi) \cong G / \operatorname{Ker}(\varphi)$.

\section{Main Results}

Theorem 3.1 Let $G_{1}$ and $G_{2}$ be groups and let $\varphi \in \operatorname{Hom}\left(G_{1}, G_{2}\right)$. Then $\varphi$ is dual homomorphism if and only if $G_{1}^{\varphi} \triangleleft G_{2}$ and $G_{2} / G_{1}^{\varphi} \cong \operatorname{Ker}(\varphi)$.

Proof Suppose $\varphi$ is dual homomorphism, then these exists $\beta \in \operatorname{Hom}\left(G_{2}, G_{1}\right)$ such that $\operatorname{Ker}(\varphi)=G_{2}{ }^{\beta}$ and $\operatorname{Ker}(\beta)=G_{1}{ }^{\varphi}$. Moreover, $\operatorname{Ker}(\beta) \triangleleft G_{2}$, we get $G_{1}^{\varphi} \triangleleft G_{2}$. By Lemma 2.8, we have $G_{2} / G_{1}^{\varphi}=G_{2} / \operatorname{Ker}(\beta) \cong G_{2}{ }^{\beta} \cong \operatorname{Ker}(\varphi)$, thus $G_{2} / G_{1}^{\varphi} \cong \operatorname{Ker}(\varphi)$.

Conversely, suppose that $G_{1}^{\varphi} \triangleleft G_{2}$ and $G_{2} / G_{1}^{\varphi} \cong \operatorname{Ker}(\varphi)$, then there exists an isomorphism $f: G_{2} / G_{1}^{\varphi} \rightarrow \operatorname{Ker}(\varphi)$. Let $\beta: G_{2} \rightarrow G_{1}\left(g^{\beta}=(\bar{g})^{f}\right.$, for every $\left.g \in G_{2}, \bar{g}=g G_{1}^{\varphi}\right)$. It is easy to prove that $\beta$ is well defined. Since $f$ is an isomorphism, it is obvious that $(g h)^{\beta}=(\overline{g h})^{f}=\left(g h G_{1}^{\varphi}\right)^{f}=\left(g G_{1}^{\varphi}\right)^{f}\left(h G_{1}^{\varphi}\right)^{f}=g^{\beta} h^{\beta}$ for arbitrary $g, h \in G_{2}$, then $\beta \in \operatorname{Hom}\left(G_{2}, G_{1}\right)$. Furthermore, we have $G_{2}^{\beta}=\left(G_{2} / G_{1}^{\varphi}\right)^{f}=\operatorname{Ker}(\varphi)$ and

$$
\operatorname{Ker}(\beta)=\left\{g \in G_{2} \mid g^{\beta}=1\right\}=\left\{g \in G_{2} \mid\left(g G_{1}^{\varphi}\right)^{f}=1\right\}=\left\{g \in G_{2} \mid g G_{1}^{\varphi}=G_{1}^{\varphi}\right\}=G_{1}^{\varphi}
$$

Hence, $\varphi$ is dual homomorphism by Definition 2.4 .

Corollary 3.2 Let $G_{1}$ and $G_{2}$ be groups, $\varphi \in \operatorname{Hom}\left(G_{1}, G_{2}\right)$. Then the following two statements are equivalent.

(1) $\varphi$ is dual homomorphism.

(2) These exists $\beta \in \operatorname{Hom}\left(G_{2}, G_{1}\right)$ such that $\operatorname{Ker}(\varphi) \cong G_{2}{ }^{\beta}$ and $\operatorname{Ker}(\beta)=G_{1}{ }^{\varphi}$.

Proof Suppose $\varphi$ is dual homomorphism, then these exists $\beta \in \operatorname{Hom}\left(G_{2}, G_{1}\right)$ such that $\operatorname{Ker}(\varphi)=G_{2}{ }^{\beta}$ and $\operatorname{Ker}(\beta)=G_{1}^{\varphi}$. It is clear that $\operatorname{Ker}(\varphi) \cong G_{2}{ }^{\beta}$ and $\operatorname{Ker}(\beta)=G_{1}^{\varphi}$.

Conversely, if there exists $\beta \in \operatorname{Hom}\left(G_{2}, G_{1}\right)$ such that $\operatorname{Ker}(\varphi) \cong G_{2}{ }^{\beta}$ and $\operatorname{Ker}(\beta)=G_{1}^{\varphi}$. It is obvious that $G_{1}^{\varphi} \triangleleft G_{2}$. Moreover, it is enough to show that $G_{2} / G_{1}^{\varphi}=G_{2} / \operatorname{Ker}(\beta) \cong G_{2}{ }^{\beta} \cong \operatorname{Ker}(\varphi)$ by Lemma 2.8. Then $\varphi$ is dual homomorphism following Theorem 3.1.

Corollary 3.3 Let $G_{1}$ and $G_{2}$ be groups and let $\alpha \in I s o\left(G_{1}, G_{2}\right)$. Then $\alpha$ is dual homomorphism.

Proof Since $\alpha \in \operatorname{Iso}\left(G_{1}, G_{2}\right), \operatorname{Ker}(\alpha)=1$ and $G_{1}^{\alpha}=G_{2}$. Thus $G_{1}^{\varphi} \triangleleft G_{2}$ and $G_{2} / G_{1}^{\varphi} \cong \operatorname{Ker}(\varphi)$. According to Theorem 3.1, $\alpha$ is dual homomorphism.

Theorem 3.4 The composition of dual homomorphism is not necessary dual homomorphism.

Proof Let $G=C_{2} \times C_{4}$, write $C_{2}=\langle x\rangle$ and $C_{4}=<y>$. Suppose that $\pi: G \rightarrow G$ 
$\left((x, y)^{\pi}=(x, 1)\right)$ and $\gamma: G \rightarrow G\left((x, y)^{\gamma}=\left(y^{\theta}, x^{\sigma}\right)\right)$ are distinct homomorphisms of $G$, where $\theta: C_{4} \rightarrow C_{2}$ by $\left(y^{k}\right)^{\theta}=x^{k}$ and $\sigma: C_{2} \rightarrow C_{4}$ by $\left(x^{k}\right)^{\sigma}=y^{2 k}$ are homomorphisms. It is obvious that $G^{\pi} \triangleleft G$ and $G / G^{\pi} \cong C_{4} \cong \operatorname{Ker}(\pi)$, then $\pi$ is dual homomorphism. Similarly, it is easy to get $G^{\gamma} \triangleleft G$ and $G / G^{\gamma} \cong C_{2} \cong \operatorname{Ker}(\gamma)$, thus $\gamma$ is dual homomorphism. Note that $\pi \gamma: G \rightarrow G\left((x, y)^{\pi /}=\left(1^{\theta}, x^{\sigma}\right)=\left(1, x^{\sigma}\right)\right)$ is well defined by the definition of $\pi$ and $\gamma$. Therefore $G^{\pi \gamma}=1 \times C_{2} \triangleleft G$ and $\operatorname{Ker}(\pi \gamma)=\left\{(x, y) \in G \mid(x, y)^{\pi /}=1\right\}=\left\{(x, y) \in G \mid\left(1, x^{\sigma}\right)=1\right\}=1 \times C_{4}$.

Since $G / G^{\pi \gamma} \cong C_{2} \times C_{2}$ and $\operatorname{Ker}(\pi \gamma)=1 \times C_{4}, G / G^{\pi \gamma}$ is not isomorphic to $\operatorname{Ker}(\pi \gamma)$. Hence, $\pi \gamma$ is not dual homomorphism.

Corollary 3.5 Let $G_{1}, G_{2}, G_{3}$ be groups. Suppose that $\varphi \in \operatorname{Hom}\left(G_{1}, G_{2}\right)$ is a dual homomorphism and $\psi \in \operatorname{Hom}\left(G_{2}, G_{3}\right)$ is an isomorphism. Deduce that $\varphi \psi$ is a dual homomorphism of $G_{1}$ to $G_{3}$. In particular, if $\psi \in \operatorname{Aut}\left(G_{2}\right)$, then $\varphi \psi$ is a dual homomorphism of $G_{1}$ to $G_{2}$.

Proof Since $\varphi \in \operatorname{Hom}\left(G_{1}, G_{2}\right)$ is a dual homomorphism, it is clear that $G_{1}^{\varphi} \triangleleft G_{2}$ and $G_{2} / G_{1}^{\varphi} \cong \operatorname{Ker}(\varphi)$. Because $\psi$ is an isomorphism, it is enough to show that $\varphi \psi \in \operatorname{Hom}\left(G_{1}, G_{3}\right)$ and $\left(G_{1}^{\varphi}\right)^{\psi}=G_{1}^{\varphi \psi} \triangleleft G_{2}^{\psi}=G_{3}$. Following Lemma 2.7 and $\psi$ is an isomorphism, then $G_{3} / G_{1}^{\varphi \psi} \cong G_{3} / G_{1}^{\varphi} \cong G_{2} / G_{1}^{\varphi} \cong \operatorname{Ker}(\varphi)$. Now we just prove that $\operatorname{Ker}(\varphi)=\operatorname{Ker}(\varphi \psi)$. Actually, for arbitrary $x \in \operatorname{Ker}(\varphi), x^{\varphi}=1$. So $x^{\varphi \psi}=1^{\psi}=1$, hence $x \in \operatorname{Ker}(\varphi \psi)$, thus $\operatorname{Ker}(\varphi) \subseteq \operatorname{Ker}(\varphi \psi)$. Similarly, we have $y^{\varphi \psi}=1$ for arbitrary $y \in \operatorname{Ker}(\varphi \psi)$, then $y^{\varphi \psi}=y^{\varphi}=1, y \in \operatorname{Ker}(\varphi)$, thus $\operatorname{Ker}(\varphi) \supseteq \operatorname{Ker}(\varphi \psi)$. We have $G_{3} / G_{1}^{\varphi \psi} \cong \operatorname{Ker}(\varphi)=\operatorname{Ker}(\varphi \psi)$. By Theorem 3.1, $\varphi \psi$ is a dual homomorphism of $G_{1}$ to $G_{3}$.

In particular, if $\psi \in \operatorname{Aut}\left(G_{2}\right)$, let $G_{3}=G_{2}$. Similarly, we get that $\varphi \psi$ is a dual homomorphism of $G_{1}$ to $G_{2}$.

Theorem 3.6 Let $G_{1}, G_{2}, H_{1}, H_{2}$ be groups. Moreover, suppose that $G_{1} \cong G_{2}$ and $H_{1} \cong H_{2}$. If $G_{1}$ and $H_{1}$ are dual, then $G_{2}$ and $H_{2}$ are dual.

Proof Let $\varphi: G_{1} \rightarrow G_{2}$ and $\psi: H_{1} \rightarrow H_{2}$ be distinct isomorphisms. Suppose that $\beta$ is a normal homomorphism of $G_{2}$ to $H_{2}$. We will prove that $H_{2} / G_{2}{ }^{\beta} \cong \operatorname{Ker}(\beta)$. Obviously, $\varphi \beta \psi^{-1} \in \operatorname{Hom}\left(G_{1}, H_{1}\right)$. Since $G_{1}$ and $H_{1}$ are dual, it follows that $G_{1}^{\varphi \beta \psi^{-1}} \triangleleft H_{1}$ and $H_{1} / G_{1}^{\varphi \beta \psi^{-1}}$ $\cong \operatorname{Ker}\left(\varphi \beta \psi^{-1}\right)$.

Let $\theta: H_{1} \rightarrow \mathrm{H}_{2} / \mathrm{G}_{2}{ }^{\beta}\left(x^{\theta}=x^{\psi} G_{2}{ }^{\beta}, \quad x \in H_{1}\right)$. Then $\theta$ is well defined, and it is easy to check $\theta$ is a surjective homomorphism. By Lemma $2.8, H_{1} / \operatorname{Ker}(\theta) \cong H_{2} / G_{2}{ }^{\beta}$ and

$$
\operatorname{Ker}(\theta)=\left\{x \in H_{1} \mid x^{\theta}=1\right\}=\left\{x \in H_{1} \mid x^{\psi} G_{2}{ }^{\beta}=G_{2}^{\beta}\right\}=\left\{x \in H_{1} \mid x \in G_{2}^{\beta \psi^{-1}}\right\}=G_{1}^{\phi \beta \psi^{-1}}
$$

Thus $H_{2} / G_{2}{ }^{\beta} \cong H_{1} / \operatorname{Ker}(\theta)=H_{1} / G_{1}^{\varphi \beta \psi^{-1}} \cong \operatorname{Ker}\left(\varphi \beta \psi^{-1}\right)$.

Let $\Phi: \operatorname{Ker}\left(\varphi \beta \psi^{-1}\right) \rightarrow \operatorname{Ker}(\beta)$, where $x^{\Phi}=x^{\varphi}$. For any $x \in \operatorname{Ker}\left(\varphi \beta \psi^{-1}\right), x^{\varphi \beta \psi^{-1}}=1$. Since $\psi$ is isomorphism, $x^{\varphi \beta}=1$. We obtain $x^{\varphi} \in \operatorname{Ker}(\beta)$, i.e. $\Phi$ is well defined. For arbitrary $x_{1}, x_{2} \in \operatorname{Ker}\left(\varphi \beta \psi^{-1}\right)$, since $\varphi$ is isomorphism, we have $\left(x_{1} x_{2}\right)^{\Phi}=\left(x_{1} x_{2}\right)^{\varphi}=x_{1}^{\varphi} x_{2}{ }^{\varphi}=x_{1}^{\Phi} x_{2}{ }^{\Phi}$. Hence, $\Phi$ is homomorphism. For any $y \in \operatorname{Ker}(\beta) \leq G_{2}$, there exists $x \in G_{1}$ such that $x^{\varphi}=y$. Since $x^{\varphi \beta \psi^{-1}}=\left(\left(x^{\varphi}\right)^{\beta}\right)^{\psi^{-1}}=\left(y^{\beta}\right)^{\psi^{-1}}=1^{\psi^{-1}}=1$, it follows that $x \in \operatorname{Ker}\left(\varphi \beta \psi^{-1}\right)$. So $\Phi$ is surjective. Furthermore, $\operatorname{Ker}(\Phi)=\left\{x \in \operatorname{Ker}\left(\varphi \beta \psi^{-1}\right) \mid x^{\Phi}=x^{\varphi}\right\}=1$. So we have that $\Phi$ is injective. Therefore 
$\Phi$ is an isomorphism. Thus $H_{2} / G_{2}{ }^{\beta} \cong \operatorname{Ker}(\beta)$, i.e. $G_{2}$ and $H_{2}$ are dual.

The following corollary identifies a direct result of theorem 3.6.

Corollary 3.7 Let $G_{1}$ and $G_{2}$ be two isomorphic groups. If $G_{1}$ is morphic group so is $G_{2}$.

\section{References}

[1] W. K. Nicholson and E. Sánchez Campos, Rings with the dual of the isomorphism theorem, J. Algebra, 271(2004) 391-406.

[2] Y. Li, W. K. Nicholson, L. Zan, Morphic groups, J. Pure Appl. Algebra, 214 (2010) 1827-1834.

[3]Q. Wang, K. Long and L. Feng, The Quasi-Morphic property of group. Bulletin of the Iranian Mathermatical Society, Vol. 39, No. 1 (2013) 175-185.

[4] J. S. Rose, A Course on Group Theory. Cambridge University Press, Cambridge, 1978.

[5] R. Kellil, Some propreties on morphic groups. Pure Mathematical Sciences,

Vol. 2, No. 2 (2013) 55-68.

[6]W. R. Scott. Group Theory. Prentice-Hall, 1964.

[7] Y. Li and W. K. Nicholson, Ehrlich's theorem for groups, Bull. Austral. Math. Soc. 81 (2010) 304-309. 